\title{
A MODEL OF OFDM BASED MARITIME VHF COMMUNICATION SYSTEM FOR DATA EXCHANGE
}

\author{
Sanjin Valčić ${ }^{1}$ \\ Tibor Pogány ${ }^{1,2}$ \\ Zoran Mrak ${ }^{1}$ \\ ${ }^{1}$ Faculty of Maritime Studies Rijeka, University of Rijeka, Croatia \\ ${ }^{2}$ John von Neumann Faculty of Informatics, Institute of Applied Mathematics, Óbuda University, Budapest, Hungary
}

\begin{abstract}
In the maritime Very High Frequency (VHF) band, there are no systems for transmitting large amounts of data. Therefore, it is necessary to develop new systems that would modernize the Global Maritime Distress and Safety System (GMDSS), significantly relieve the Automatic Identification System's (AIS) communication channels, and set guidelines for the development of communication infrastructure of the e-Navigation. In line with this, analytical and simulation models of the maritime VHF data transmission communication system using Orthogonal Frequency Division Multiplexing (OFDM) modulation are worked out in this paper. The achieved data rate, the spectral efficiency and the bit error rate (BER) represent validation parameters on which the results of the analytical and simulation models are evaluated. It is concluded that the application of the digital OFDM modulation in the maritime VHF band may improve the GMDSS system by achieving higher data rates compared to the current terrestrial mandatory systems for data exchange, i.e. Digital Selective Calling (DSC) and AIS.
\end{abstract}

Keywords: Maritime VHF communications; OFDM modulation; AWGN; SNR; BER

\section{INTRODUCTION}

The Inmarsat satellite communication system provides with more ways of communication in relation to maritime terrestrial communications. Namely, it enables digital voice communications, exchange of large amounts of data, as well as the Internet Protocol (IP) connection. These features, as well as the ease of operation, are the main reasons for the more frequent use of Inmarsat devices on board ships in comparison to the terrestrial communication devices, resulting in the non-utilization of a large portion of the frequency spectrum allocated for terrestrial communications. However, the broad range of possibilities implies a high service price. In addition, Inmarsat satellites do not cover the areas of the Earth's poles. On the other hand, amongst the maritime terrestrial communication systems, the VHF systems are the most common form of communication between ships at short distances, as well as between ships and land objects in coastal and port areas. However, as the DSC is the only mandatory digital communication system in the maritime VHF band, intended for alerting and announcing further analogue communications according to the GMDSS priorities, there is a need for the development and application of new digital systems for data transmission. A system already used on ships, is the AIS, which serves to identify and track ships, based on the exchange of data between ships, and between ships and base stations by using two maritime VHF channels. Due to its technology and efficiency, the AIS became mandatory for vessels that are not under the Safety of Life at Sea (SOLAS) Convention. Additionally, the AIS is often used to send other data, such as Aids to Navigation (AtoN), Application Specific Messages (ASM), Search and Rescue Transmitter AIS (SART-AIS), Man Overboard Unit (MOB) and Emergency Position-Indicating Radio Beacon AIS (EPIRB-AIS).

In some areas, the excessive use of AIS resulted in the congestion of its channels and degradation of its efficiency [13]. 
This initialized various researches and the development of new solutions for data transmission in the maritime VHF band. In [2] the issue of applying new technologies in maritime communications has been introduced, and the research and strategy of the Norwegian MarCom project with the aim of enhancing maritime communications from the aspect of increasing their coverage and capacity, was described.

Moreover, in its recommendation [11], the International Telecommunications Union (ITU) proposed four possible systems for data transmission that could be used in the maritime VHF band. Such systems were developed and implemented in [10], [18]. Furthermore, at the World Radiocommunication Conference (WRC) held in 2012, two Resolutions [32], [33] were adopted proposing the conduction of researches and studies related to new radiocommunication systems that would modernize the GMDSS and relieve AIS channels. According to [15], some of the existing analogue channels of maritime VHF frequency band were reallocated for the AIS, as well as for testing new digital communication systems. The use of additional channels and digital VHF systems for data transmission will relieve AIS channels, modernize the GMDSS, and lay the foundations for future development and implementation of the International Maritime Organization's (IMO) and the International Association of Marine Aids to Navigation and Lighthouse Authorities' (IALA) strategy of e-Navigation. According to the definition given by the IMO, e-Navigation is harmonized collection, integration, exchange, presentation and analysis of maritime information on board and ashore, using electronic systems, in order to improve the safety of navigation and other services related to safety at sea and protection of the marine environment. Consequently, the IALA developed a technological concept called the VHF data exchange system (VDES) aimed at solving problems of AIS channels' congestion and the degradation of its efficiency. Simultaneously, the VDES allows a wide exchange of data in line with the strategy of $e$-Navigation and potentially supports the modernization of the GMDSS system [14].

One of the proposed digital communication technologies already used in land communications is the OFDM modulation. This modulation technique uses multiple parallel and separately modulated carrier waves, the subcarriers, within a specific communication channel as opposed to conventional communication systems which use a single modulated carrier wave. For this reason, a system using an OFDM modulation is spectrally efficient and achieves relatively high data rates, and thus transmits a large amount of information. Therefore, a systematic analysis of the maritime VHF communication system for data transmission, using digital OFDM modulation, through the development of analytical and simulation models of its physical layer, is provided in this paper. One of such systems is proposed in [7] for coast-to-sea and coast-to-air propagation by using an OFDM modulation, but in the Ultra High Frequency (UHF) band. Another system that uses an OFDM modulation for electronic mail exchange within the maritime High Frequency (HF) channel of $3 \mathrm{kHz}$ bandwidth is described in [12].

\section{OFDM MODULATION: AN OVERVIEW}

The first concept of the OFDM technology was presented by Robert W. Chang in [3]. He demonstrated the principle of simultaneous transmission of multiple signals within the band-limited channel, without interchannel and intersymbol interference. The OFDM enables the subcarriers' side bands to overlap without mutual interference, which is achieved by their orthogonality. In other words, the subcarriers are independent or orthogonal if the spacing between them is inversely proportional to the duration of the transmitted symbol on each of them. Due to the orthogonality, boundary or zero value of the each carrier's spectrum overlaps with the peak value or the central frequency of the adjacent carrier.

\section{MODULATION PRINCIPLE}

The OFDM modulation technique divides a communication channel of a certain bandwidth into more narrow channels, i.e. it uses multiple separate carriers for data transmission within one channel. Therefore, it is necessary to separately modulate each subcarrier for the transmission of data. In other words, digital information or the total amount of bits is divided into several series or blocks, in order to modulate multiple carrier waves. The results of digital modulations are complex symbols, $s_{k}$, for $k=0,1,2, \ldots N-1$, where $N$ is the number of so called parallel bits which corresponds to the number of subcarriers. Furthermore, these symbols will be transmitted by the OFDM modulation in the following signal form:

$$
s(t)=\sum_{k=0}^{N-1} s_{k} e^{j 2 \pi f_{k} t} \text { for } 0 \leq t \leq T_{s}
$$

where $f_{k}=f_{0}+\Delta f$ is the frequency of $k^{\text {th }}$ subcarrier, $\Delta \mathrm{f}$ is the spacing between subcarriers frequencies and $T_{s}$ is the transmitted symbol duration [26].

The implementation of the OFDM modulation is possible by using a discrete Fourier transform (DFT). Thus, in order to transmit the OFDM signal as defined by (1), under the assumption that the signal is sampled during the time interval $T_{\text {sample }}=T_{s} / N$, then the $n^{\text {th }}$ sample of the OFDM signal (where $n=k$ ) is:

$$
s_{n}=\sum_{k=0}^{N-1} s_{k} e^{j 2 \pi f_{k} \frac{n T_{s}}{N}}
$$

For simplicity reasons, the frequency of the first subcarrier is assumed to be $f_{0}=0$. As a result, the orthogonality principle is defined by $f_{k} \cdot T_{s}=\mathrm{k}$ and each sample by:

$$
s_{n}=\sum_{k=0}^{N-1} s_{k} e^{j \frac{2 \pi n k}{N}}=\operatorname{IDFT}\left\{s_{k}\right\}
$$

where the IDFT is the inverse discrete Fourier transform. Analogously to the IDFT at the transmitter, the receiver uses the DFT of the received signal in order to successfully demodulate transmitted symbols. 


\section{CYCLIC EXTENSION}

After performing an IDFT, a cyclic extension is added to the obtained signal in order to avoid intersymbol interference caused by the dispersion of the communication channel. Since some of the carrier waves may be received with a certain time delay, due to different propagation conditions in the communication channels, the interference between adjacent symbols, as well as between the adjacent subcarriers, may occur in the OFDM receivers [6], [24], [30]. There are three ways of inserting a cyclic extension: cyclic prefix, cyclic suffix and their combination. Thus, the cyclic extension is achieved by copying a part of OFDM signal and inserting it at the beginning and/ or the end of the OFDM signal, which ensures the periodicity of the signal, i.e. the preservation of orthogonality between subcarriers. If the $T_{g}$ indicates the duration of the cyclic extension added to the OFDM signal, then the total duration of the OFDM symbol extends to $T=T_{s}+T_{g}$.

\section{ADVANTAGES AND DISADVANTAGES OF OFDM MODULATION}

One of the key advantages of the OFDM modulation in comparison to single carrier systems is the robustness to intersymbol and interchannel interference. Namely, the OFDM symbol duration is much longer than the symbol interval of the equivalent single carrier system, and thus, the interference can only affect a small portion of the OFDM symbols. This results in a simpler implementation of the OFDM receiver as opposed to one used in a single carrier system. The second advantage of the OFDM modulation is the resistance to fading caused by multipath propagation. Thus, in contrast to single carrier communication systems, where the multipath propagation affects the whole signal, i.e. the complete information, the OFDM modulation divides a communication channel into several narrowband channels resistant to multipath propagation. The duration of the OFDM symbol is increased by turning one channel with high data rate into several parallel channels with lower data rates, reducing the relative delay spread in the channel. Furthermore, the cyclic extension cancels the intersymbol interference, and enables easier synchronization between the receiver and the transmitter. Another advantage of the OFDM modulation is the high spectral efficiency. Namely, unlike the single carrier systems which require guard bands inside the channels of a certain bandwidth, the OFDM modulation very effectively exploits the allowed bandwidth of the communication channel as the subcarriers overlap [6], [24], [30], [31].

However, OFDM modulation has several disadvantages. The OFDM symbol is the sum of subcarriers' signals resulting in a high value of the Peak-to-Average Power Ratio (PAPR) [4], [21]. Therefore, systems with OFDM modulation must use broadband linear amplifiers. If this is not done, the peak power value of the signal would enter the nonlinear range of the power amplifier, resulting in signal distortion, intermodulation between carrier waves and power transmission outside the permitted frequency spectrum, i.e. outside the communication channel. Therefore, it is necessary to use different techniques to reduce PAPR value, which are not the subject of this research. Another disadvantage of the OFDM modulation is a distinct sensitivity to the subcarriers' frequency shift between the transmitter and the receiver. As the frequency spectrum of each carrier is only a small fraction of the communication channel's bandwidth, a very small frequency shift will cause disturbances such as attenuation and phase rotation, as well as interchannel interference. Therefore, communication systems using the OFDM modulation must have very precise estimation of the frequency shift in receivers. Moreover, the OFDM systems are very sensitive to Doppler frequency shift or spread caused by motion of the transmitter and/or receiver which results in the loss of orthogonality between carrier waves. Specifically, as the spacing between the carriers' frequencies is relatively small, the Doppler spread or shift can affect their mutual interference. If all subcarriers were exposed to a common Doppler frequency shift, it could be compensated in the receivers to avoid interchannel interference. However, if the Doppler frequency shift is in the order of spacing between the subcarriers, the interchannel interference causing extreme degradation of system performance will appear. Accordingly, the spacing between the subcarriers must be much higher than the maximum Doppler frequency shift, i.e. the following condition must be met:

$$
\frac{f_{D}}{\Delta f}=f_{D} \cdot T_{S} \ll 1
$$

where $f_{D}$ is the maximum Doppler frequency shift [6], [24], [30], [31].

\section{A MODEL OF THE MARITIME VHF OFDM DATA EXCHANGE SYSTEM}

In developing the model of a communication system which uses the digital OFDM modulation technique, special attention should be paid to its requirements, such as available channel bandwidth, acceptable delay spread in the channel, required data rate, and the characteristics of the Doppler frequency shift. These requirements result in setting up the following basic parameters for modeling communication system which uses OFDM modulation: the number of subcarriers, cyclic extension and total symbol duration, subcarriers' frequency spacing, modulation and coding methods [5], [23], [31].

When choosing the number of subcarriers it should be taken into account that a greater number of subcarriers reduces the delay spread in the communication channel, i.e. reduces the intersymbol interference, but also results in difficult signal synchronization in the receiver. Furthermore, the duration of the cyclic extension should be two to four times longer than the average delay of the communication channel. Moreover, the spacing between subcarriers must be within acceptable range due to signal synchronization in the receiver, which primarily depends on the available channel bandwidth, or the necessary 
number of subcarriers. When selecting the digital modulation technique it is also important to know the requirements of the communication system, because different modulation techniques result in different performances. Finally, the coding technique, i.e. the detection and correction of random errors generated within the communication channel has a significant role in developing the model of the communication system using OFDM modulation.

\section{MODEL OF THE MARITIME VHF OFDM TRANSMITTER}

Prior to the modulation of $N$ carrier waves, it is necessary to divide the information or the sequence of bits into $N$ blocks or parallel bits. Furthermore, digital modulation techniques are used for separate modulation, i.e. for mapping $N$ parallel bits to $N$ subcarriers in the OFDM transmitter. The digital modulation techniques are usually classified according to the parameter of the carrier wave which is modulated (amplitude, frequency, phase) and to the number of levels assigned to the modulated parameter $M$. According to [17], [20], [34], the most commonly used digital modulation techniques in OFDM systems are $M$-PSK and $M$-QAM. The techniques modulate the amplitude and/or phase of the carrier waves, leaving their frequency unchanged. If their frequency is changed, it will result in a distortion of the orthogonality between subcarriers so the proposed model of the OFDM transmitter incorporates digital amplitude and phase modulation techniques. After mapping complex symbols, the IDFT is performed and cyclic extension added. The obtained parallel data is then converted into serial data or bits which are afterwards converted into an analogue baseband signal. Finally, the VHF upconversion is performed and the passband signal is created.

In the proposed model of the OFDM transmitter 8, 16, 32 and 64 subcarriers are used, respectively. In the maritime VHF band, the communication channels' bandwidth is $B=25 \mathrm{kHz}$, including guard bands. One of the channels intended for testing new digital communication systems in the maritime VHF band is channel 24, whose carrier frequency (for ship stations) is $f_{c}=157.200 \mathrm{MHz}$. Assuming that the proposed OFDM system uses a permitted channel bandwidth of $16 \mathrm{kHz}$ ( $9 \mathrm{kHz}$ is intended for guard bands) the parameters of the OFDM modulation are calculated and shown in Tab. 1.

Tab. 1. Parameters of the OFDM modulation for the proposed system

\begin{tabular}{|c|c|c|c|c|}
\hline $\begin{array}{c}\text { Number of } \\
\text { subcarriers, } \\
N\end{array}$ & $\begin{array}{c}\text { Frequency } \\
\text { spacing, } \\
\Delta f[\mathrm{~Hz}]\end{array}$ & $\begin{array}{c}\text { Symbol } \\
\text { duration, } \\
T_{s}[\mu \mathrm{s}]\end{array}$ & $\begin{array}{c}\text { Cyclic } \\
\text { extension } \\
\text { duration, } \\
T_{g}[\mu \mathrm{s}]\end{array}$ & $\begin{array}{c}\text { Total OFDM } \\
\text { symbol } \\
\text { duration, } \\
T=T_{s}+T_{g}[\mu \mathrm{s}]\end{array}$ \\
\hline 8 & 1777.78 & 562.50 & 140.63 & 703.13 \\
\hline 16 & 941.18 & 1062.50 & 265.63 & 1328.13 \\
\hline 32 & 484.85 & 2062.50 & 515.63 & 2578.13 \\
\hline 64 & 246.15 & 4062.50 & 1015.63 & 5078.13 \\
\hline
\end{tabular}

In Tab. 1, the spacing between subcarriers is calculated according to the expression for the frequency spectrum bandwidth $B=(N+1) \cdot \Delta f$, symbol duration according to $T_{s}=1 / \Delta f$ and cyclic extension duration $T_{g}=T_{s} / 4$. The last expression for the cyclic extension duration is assumed and determined according to land wireless communication systems using OFDM modulation, i.e. Digital Audio Broadcasting (DAB), Digital Video Broadcasting (DVB) and Wireless Local Area Network (WLAN) [1], [25].

Tab. 2. Symbol rate and spectral efficiency of the proposed OFDM system model

\begin{tabular}{|c|c|c|c|}
\hline $\begin{array}{c}\text { Number of } \\
\text { subcarriers, } \\
N\end{array}$ & $\begin{array}{c}\text { Total OFDM } \\
\text { symbol } \\
\text { duration, } \\
T=T_{s}+T_{g}[\mu \mathrm{s}]\end{array}$ & $\begin{array}{c}\text { Symbol rate, } \\
R_{s} \\
{[\mathrm{ksymbol} / \mathrm{s}]}\end{array}$ & $\begin{array}{c}\text { Spectral } \\
\text { efficiency, } \\
\eta, \\
{[\text { symbol/s } / \mathrm{Hz}]}\end{array}$ \\
\hline 8 & 703.13 & 11.38 & $0.71=71 \%$ \\
\hline 16 & 1328.13 & 12.05 & $0.75=75 \%$ \\
\hline 32 & 2578.13 & 12.41 & $0.78=78 \%$ \\
\hline 64 & 5078.13 & 12.60 & $0.79=79 \%$ \\
\hline
\end{tabular}

The OFDM system with the specified parameters is robust to multipath fading in communication channels having a maximum delay spread of wave components to $\tau_{m a}=T_{g} \mu \mathrm{s}$. Symbol rate of such a system as well as its spectral efficiency are shown in Tab. 2. The symbol rates were calculated according to $R_{s}=N / T$, and the spectral efficiency according to $\eta=R_{s} / B$, where $B=16 \mathrm{kHz}$, i.e. the permitted maritime VHF channel bandwidth. It should be noted that if $M$-level digital modulations are used, where $M=2^{\mathrm{n}}$, the symbol rate and spectral efficiency are increased $n=\log _{2} M$ times, i.e. every symbol carries $n$ bits [19], [22]. Furthermore, in this paper it is assumed that the maximum relative speed between ships is $v=60 \mathrm{~km} / \mathrm{h}(\approx 30 \mathrm{kn}$.). At that speed and at the central frequency of the maritime VHF communication channel 24, the maximum Doppler frequency shift is [25]:

$$
f_{D}=\frac{v}{c} \cdot f_{C}=\frac{1}{1,080} \cdot \frac{f_{c}}{\mathrm{MHz}} \cdot \frac{v}{\mathrm{~km} \cdot \mathrm{h}^{-1}}=8.73 \mathrm{~Hz}
$$

where $c$ is the speed of light. As can be seen above, the movement between ships will not cause interchannel interference, because the spacing between subcarriers is much higher than the maximum Doppler frequency shift for all numbers of subcarriers.

\section{MODEL OF THE MARITIME VHF OFDM RECEIVER}

In the maritime VHF OFDM receiver, the reverse procedure is performed in relation to the OFDM transmitter. Thus, after receiving the signal, the downconversion is performed, i.e. the conversion of the OFDM signal from the frequency band of the maritime VHF channel 24 to the baseband. Subsequently, 
this signal is converted into a digital (sampled) signal $y_{n}$, the cyclic extension is removed and serial data is converted into $N$ parallel blocks that correspond to the number of subcarriers. After the implementation of the DFT, the output stream of samples or complex OFDM symbols is read as follows:

$$
\begin{gathered}
r_{k}=\sum_{n=0}^{N-1} y_{n} e^{-j \frac{2 \pi n k}{N}} \\
=\sum_{n=0}^{N-1}\left(h_{n} s_{n}+n_{n}\right) e^{-j \frac{2 \pi n k}{N}} \\
=h_{k} s_{k}+n_{k}, \text { for } k=n
\end{gathered}
$$

where $h_{k}$ represents the channel impulse response and $n_{k}$ additive noise to each carrier wave.

The main problem in the design and implementation of digital systems, is time synchronization between the receiver and the transmitter. As the receiver has no prior knowledge of whether there is data in the received signal or not, it is crucial to detect the moment of the receipt of the transmitted data. After the transmitted data packets or frames are detected within the OFDM signal, the receiver can eliminate the cyclic extension and implement DFT on the received signal and demodulate subcarriers. However, this process is not simple, because the transmitted signals typically have frequency and phase shifts. In most practical OFDM systems, time and frequency synchronization of the receiver and the transmitter are based on the correlation functions performed on cyclic extensions or additional reference symbols in OFDM signals.

\section{MODEL OF THE MARITIME VHF CHANNEL}

In general, communication channels in the maritime VHF band can be described by using multipath fading characteristics. This is a phenomenon which occurs when radio waves reach the receiving antenna from different directions with random amplitudes, frequencies and phases, causing major changes in the phases of the incident radio wave components. Basically, fading can be divided into slow and fast fading (Simon and Alouini, 2000; Schulze and Lüders, 2005). The difference between slow and fast fading is important for mathematical modeling of communication channels with such features and for the performance evaluation of communication systems using such channels. This term refers to coherence time of channels $T_{c}$, representing the period during which the process of fading correlates. Furthermore, coherence time is associated with the Doppler spread, i.e. the maximum Doppler frequency shift according to $T_{c}=1 / f_{D}$ [25], [29]. If symbol duration is less than the coherence time, fading is considered slow and vice versa. Previously, it was calculated that the maximum Doppler frequency shift under relative movement between ships at a speed of $v=60 \mathrm{~km} / \mathrm{h}(\approx 30 \mathrm{kn}$.) and at a central frequency of the maritime VHF communication channel 24 is $f_{D}=8.73 \mathrm{~Hz}$. Thus, a coherence time of this channel is $T_{c}=114.55 \mathrm{~ms}$, which is far larger than the total symbol duration of the proposed model of the maritime VHF OFDM communication system. Furthermore, longer time delays of the radio wave components cause a higher frequency selectivity of the channel, i.e. the occurrence of fading stochastically influences the radio waves> frequencies. This delay is associated with a communication channelss coherence bandwidth $f_{\text {coh }}$, representing the frequency range within which the process of fading correlates. Moreover, a channel >s coherence bandwidth is associated with the maximum delay spread, according to $f_{\text {coh }}=1 / \tau_{\text {max }}$ [25], [29]. If the frequency spectrum of the transmitted signal is much smaller than the coherence bandwidth, fading is considered to be frequency non-selective or «flat» and vice versa. Assuming that the maximum delay spread is equal to the cyclic extension duration, the channel/s coherence bandwidth of the proposed model of the maritime VHF OFDM communication system is determined and shown in Tab. 3 .

Tab. 3. Coherence bandwidth of the maritime VHF communication channel

\begin{tabular}{|c|c|c|c|}
\hline $\begin{array}{c}\text { Number of } \\
\text { subcarriers, } \\
N\end{array}$ & $\begin{array}{c}\text { Subcarriers' } \\
\text { spacing, } \Delta f \\
{[\mathrm{~Hz}]}\end{array}$ & $\begin{array}{c}\text { Maximum } \\
\text { delay spread, } \\
\tau_{\max }[\mu \mathrm{s}]\end{array}$ & $\begin{array}{c}\text { Coherence } \\
\text { bandwidth, } \\
f_{\text {coh }}[\mathrm{Hz}]\end{array}$ \\
\hline 8 & 1777.78 & 140.63 & 7111.11 \\
\hline 16 & 941.18 & 265.63 & 3764.71 \\
\hline 32 & 484.85 & 515.63 & 1939.39 \\
\hline 64 & 246.15 & 1015.63 & 984.62 \\
\hline
\end{tabular}

Tab. 3 shows that the fading in OFDM communication systems is frequency non-selective, since the coherence bandwidth is greater than the bandwidth of each subcarriers channel, i.e. subcarriers> spacing. On the other hand, if single carrier communication systems are used in maritime VHF communication channels with the specified maximum delay spreads within 25 or $16 \mathrm{kHz}$ bandwidth, then they are negatively affected by the the frequency selective fading because the coherence bandwidth is less than the bandwidth of the maritime VHF channel.

When fading affects the communication channels of small bandwidth, the received signal or amplitude of the carrier wave is modulated only by the fading amplitude $\alpha$, which is represented by a random variable. Furthermore, after passing through a communication channel with the fading characteristics, the signal is further subjected to the influence of additive white Gaussian noise (AWGN) at the receiver. It is usually assumed that AWGN is statistically independent of fading amplitude and is described by spectral power density. Radio waves of higher frequency bands (VHF, UHF, SHF, etc.) propagate spatially and can reach the receiver by a direct line or by reflection, i.e. multipath propagation [9], [28]. Therefore, the modeling of communication channels with non-selective fading due to multipath propagation depends on the propagation paths of radio waves. Thus, the Rayleigh probability distribution is commonly used in communication systems in which there is no direct line of propagation of radio waves. On the other hand, the Nakagami- $n$ probability distribution, also called Rice probability distribution, is commonly used in communication systems in which there 
is a strong direct line of propagation of radio waves and many weaker multipath components [29]. In this paper, the worst case scenario is assumed, i.e. the VHF communication channel is modeled by the Rayleigh multipath fading. In this case, the probability density function of the fading amplitude in the channel is described by the following expression:

$$
p_{\alpha}(\alpha)=\frac{2 \alpha}{\alpha^{2}} e^{-\frac{\alpha^{2}}{\alpha^{2}}}, \alpha \geq 0
$$

where $\overline{\alpha^{2}}$ is the mean square value of the fading amplitude.

\section{SIMULATION MODEL RESULTS}

In digital communication systems, i.e. in their transmitters and receivers, discrete signals are analyzed and processed, while signals in the communication channels are continuous. For the purpose of the simulation model development of the proposed VHF OFDM system, the Simulink software package was used. However, as the Simulink processes signals in discrete time, the proposed simulation model of the maritime VHF communication system with OFDM modulation was generated and analyzed in the baseband frequency range of discrete signals. In other words, time-continuous signals in communication channels are presented and processed as time-discrete signals. Therefore, the conversion of the signal to the maritime VHF frequency range is not required because the relevant features can be processed and analyzed in the baseband frequency range, if the impacts within the VHF communication channels are taken into account [8], [16], [27]. Previously, it was outlined that the received signals in the receiver must be time and frequency synchronized with the transmitter. Since synchronization algorithms are not the object of this research, an ideal time and frequency synchronization of transmitters and receivers for the purpose of the simulation model, is assumed. Fig. 2 shows the basic simulation model of the proposed maritime VHF communication system for data transmission based on OFDM modulation.

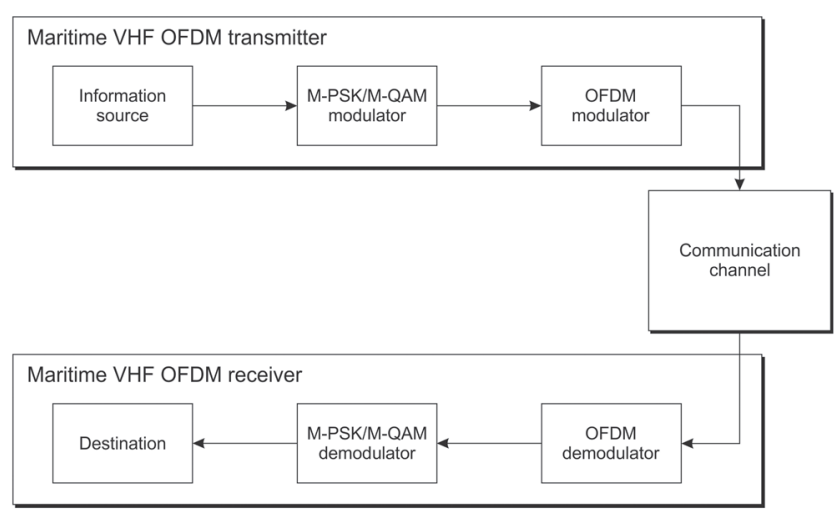

Fig. 2. Simulation model of the proposed maritime VHF OFDM communication system for data transmission
The criteria for evaluating the performance of the simulation and analytical model of the maritime VHF OFDM system are: achieved data rate, spectral efficiency and the bit error rate or probability on the receiving side of the system at different values of signal to noise ratio (SNR) expressed in dB. By using different modulation levels $M$, and number of subcarriers $N$, various data rates and spectral efficiencies of the proposed OFDM VHF communication system for data transmission were achieved and presented in Tab. 4.

Tab. 4. Data rates and spectral efficiencies of the VHF OFDM system using M-PSK or M-QAM modulations

\begin{tabular}{|c|c|c|c|c|}
\hline \multicolumn{5}{|c|}{ Data rate, $R_{b}[\mathrm{kbit} / \mathrm{s}]$} \\
\hline $\mathrm{M} N \mathrm{~N}$ & 8 & 16 & 32 & 64 \\
\hline 4 & 22.76 & 24.09 & 24.82 & 25.21 \\
\hline 8 & 34.13 & 36.14 & 37.24 & 37.81 \\
\hline 16 & 45.51 & 48.19 & 49.65 & 50.41 \\
\hline 32 & 56.89 & 60.24 & 62.06 & 63.02 \\
\hline 64 & 68.27 & 72.28 & 74.47 & 75.62 \\
\hline \multicolumn{5}{|c|}{ Spectral efficiency, $\eta[\mathrm{bit} / \mathrm{s} / \mathrm{Hz}]$} \\
\hline$M$ & 8 & 16 & 32 & 64 \\
\hline 4 & 1.42 & 1.51 & 1.55 & 1.58 \\
\hline 8 & 2.13 & 2.26 & 2.33 & 2.36 \\
\hline 16 & 2.84 & 3.01 & 3.10 & 3.15 \\
\hline 32 & 3.56 & 3.76 & 3.88 & 3.94 \\
\hline 64 & 4.27 & 4.52 & 4.65 & 4.73 \\
\hline
\end{tabular}

After determining the achieved data rates and spectral efficiencies of the maritime VHF OFDM system, it is necessary to determine the most important parameter for evaluating the results of the simulation model, i.e. the bit error rate or probability. The effect of the frequency non-selective communication channel with Rayleigh fading and the AWGN, defined by the SNR, was examined in this paper. If the SNR value is very low $(0 \mathrm{~dB})$, then the communication channel is extremely noisy, and vice versa. Fig. 3, 4, 5 and 6 show the results of simulations, i.e. the BER values in relation to SNR values in the communication channel with Rayleigh fading.

The simulations were performed at certain SNR values in the range of $0 \mathrm{~dB}$ to $60 \mathrm{~dB}$. The obtained values of BER, corresponding to SNR values, are marked with different markers, while the curves which connect them were calculated by the interpolation. The values of BER are shown by using logarithmic scale ranging from $10^{-6}$ to $10^{\circ}$, which actually show the number of erroneous bits in the maritime VHF OFDM receiver in relation to the transmitted $10^{6}$ bits.

Fig. 3 shows the simulation results of the proposed VHF OFDM system using 8 subcarriers. It can be seen that the BER parameter 
depends on the type and level of the modulation technique. The 4-PSK and the 4-QAM modulations yield almost the same performance which is also the best performance when compared to other modulation levels. In other words, at low values of SNR, i.e. in noisy communication channels, these modulations achieve minimum values of BER. The maximum values of BER appear with a 64-QAM modulation. However, at higher values of SNR (> $40 \mathrm{~dB}$ ) the 4-QAM modulation has slightly better performance, i.e. lower values of BER than the 4-PSK modulation. This is the result of the difference in the modulation principle. Namely, the PSK modulation modulates only the phase of the subcarrier while its amplitude remains constant. On the other hand, the QAM modulation simultaneously modulates the amplitude and the phase of the subcarrier. Thus, the amplitude of the QAM modulated signals depends on the information bits. Furthermore, as it was previously stated that the fading affects only the amplitude of the carrier waves, the QAM modulation is therefore more robust to it when compared to the PSK modulation.

Moreover, it is evident that QAM modulations with higher levels $(M>4)$ achieve very high values of BER. In addition, it is evident that the values of BER are constant after about $20 \mathrm{~dB}$ SNR. In other words, the gradual reduction of noise in the communication channel does not change the value of the BER, i.e. the noise has no impact on the OFDM signal. Therefore, it can be concluded that the amplitude of Rayleigh fading has a negative effect on high-level QAM modulations in the proposed VHF OFDM system. Hence, the PSK modulation shows better characteristics in the proposed maritime VHF OFDM system with 8 subcarriers within the communication channel with a Rayleigh fading and the AWGN, than the QAM modulation.

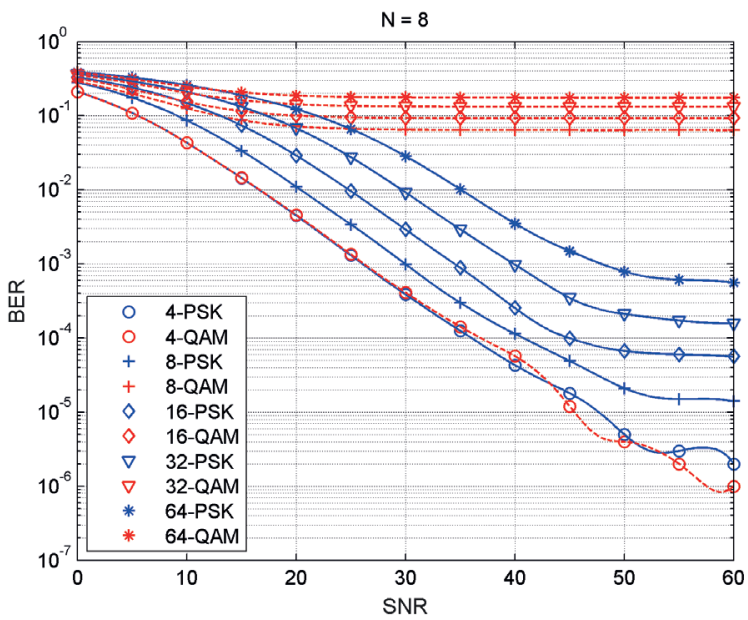

Fig. 3. The values of BER at certain $S N R$ values for $N=8$

It can also be seen that the BER parameter depends on the type and level of the modulation technique when the proposed VHF OFDM system uses more than 8 subcarriers, which is shown on Fig. 4, 5 and 6. Analogously, it can be seen that the PSK modulation performs better than the QAM modulation when $M>4$. The 4-PSK and the 4-QAM modulations again show almost identical and, when compared with other levels of modulation, the best characteristics. Again, at higher values of SNR ( $>40 \mathrm{~dB})$ the 4-QAM modulation has slightly better performance, i.e. lower values of BER than the 4-PSK modulation, as it was explained above. It is also evident that QAM modulations with higher levels have very high values of BER, and that noise reduction in the communication channel again does not change its values, i.e. it remains constant. Hence, when using larger number of subcarriers, the amplitude of Rayleigh fading also has a negative impact on high-level QAM modulations.

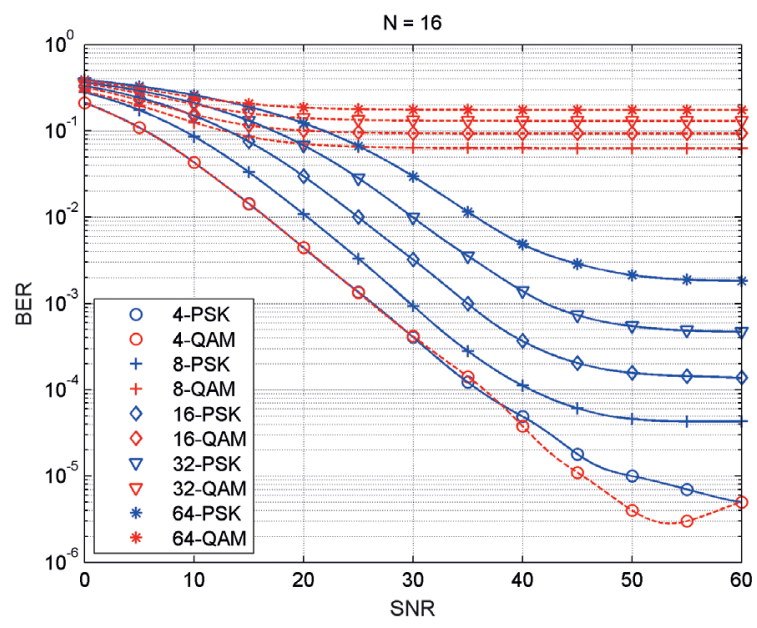

Fig. 4. The values of BER at certain $S N R$ values for $N=16$

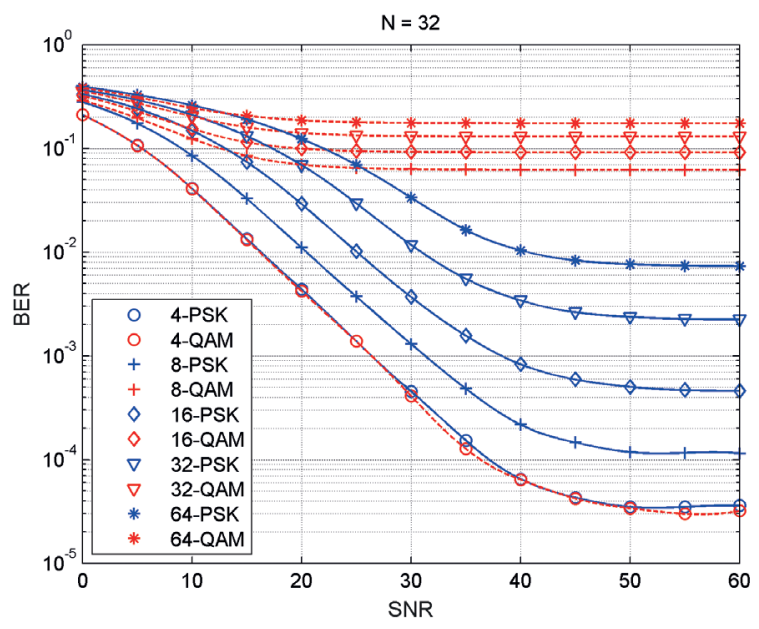

Fig. 5. The values of BER at certain $S N R$ values for $N=32$

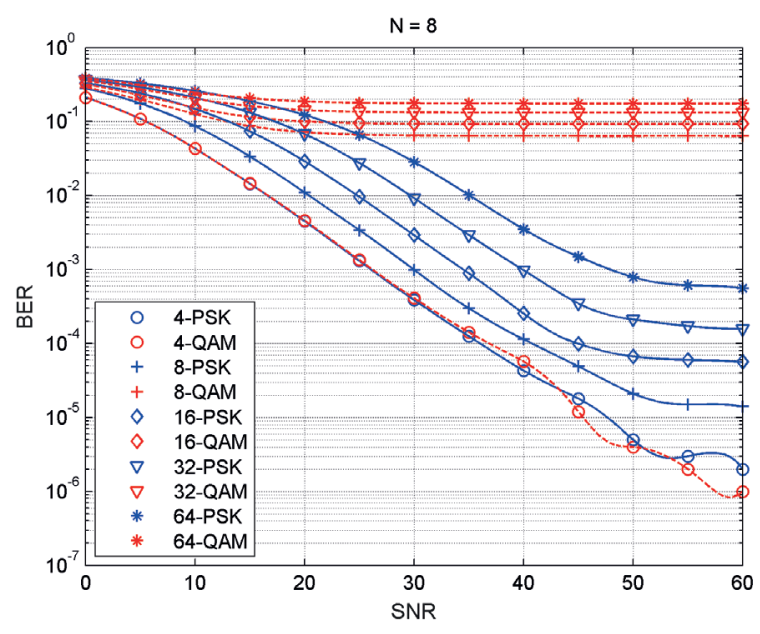

Fig. 6. The values of BER at certain $S N R$ values for $N=64$ 
However, when comparing Fig. 3, 4, 5 and 6, it is evident that the BER parameter increases with the increase in the number of subcarriers at the same SNR values. Furthermore, it can be seen that in systems with more than 8 subcarriers and at higher SNR values, the BER parameter becomes constant, which means that noise has no impact on the OFDM signal. In addition, this constant value depends on the number of subcarriers, i.e. it increases with their increase. Therefore, as noise has no impact on the BER performance of the proposed VHF OFDM system, it can be concluded that the intensity of the negative effect of the amplitude of Rayleigh fading depends on the number of subcarriers.

\section{CONCLUSIONS}

The different aspects of the maritime VHF communication problems, relevant for improving the GMDSS and relieving the AIS, as well as setting new guidelines for the implementation of $e$-Navigation were analysed in this paper. The purpose of this research was to perform a systematic analysis of the maritime VHF communication system using a digital OFDM modulation. This analysis was made based on the analytical and simulation models of the physical layer of the maritime VHF OFDM transmitter, receiver and communication channel. The proposed maritime VHF OFDM communication system for data transmission uses a different number of subcarriers with different levels of digital PSK and QAM modulations. The analytical model has shown that by using 8 subcarriers with 4-PSK and/or 4-QAM modulation within the maritime VHF communication channel of $16 \mathrm{kHz}$ bandwidth, the proposed system achieves 2.37 times higher data rate and spectral efficiency than the AIS, whose data rate is 9.6 $\mathrm{kbit} / \mathrm{s}$. Furthermore, if the proposed system uses 64 subcarriers with a 64-PSK and/or 64-QAM modulation, the achieved data rate and spectral efficiency are 7.88 times higher than the AIS. Therefore, if the proposed VHF OFDM communication system is used to transmit additional AIS data, it is possible to unload the AIS communication channels. Moreover, when the proposed system is compared to the VHF DSC, whose data rate is only $1.2 \mathrm{kbit} / \mathrm{s}$, the achieved data rate and spectral efficiency are 18.96 and 63.04 times higher, when using 4 and 64 level modulations, respectively. Since the proposed VHF OFDM communication system has better performance in terms of data rate and spectral efficiency in comparison to the AIS and VHF DSC, the implementation of this system will enable the setting of guidelines for future development of the communication infrastructure of $e$-Navigation. Moreover, such a system could be used as a supplement, or a replacement for satellite communication systems for data transmission in areas without satellite coverage. The obtained results show that the application of digital OFDM modulation in maritime VHF communications can improve the existing communication systems in GMDSS by achieving higher data rates, and thus transferring larger amounts of data.

The proposed system could be used in addition to systems for the reception of maritime safety information on board ships, and also for transmission of messages related to safety of navigation. Due to higher data rates, the proposed system could be used to transmit various navigational and meteorological warnings and reports. Furthermore, this system could also be used to update Electronic Navigational Charts (ENC) of Electronic Chart Display and Information System (ECDIS), as well as to transmit data related to maritime traffic control, various notifications to and from inland centres, as well as data related to remote maintenance. Moreover, such a system could be used for commercial purposes, both for crew members and passengers, in a way to enable connection by the Internet Protocol in areas covered by VHF coast stations.

In the development and analysis of the simulation model of the maritime VHF OFDM system, a data encryption, by which it is possible to detect and/or correct the erroneous bits, as well as the estimator of the impulse response of the communication channel in the receiver, were not used. Namely, the aforementioned techniques can achieve better system performance, i.e. lower values of bit error rate or probability but with a decrease in data rates and spectral efficiencies of the system due to the use of redundant symbols.

\section{ACKNOWLEDGEMENT}

The authors acknowledge the support of the research project "Information-communication technologies in intelligent traffic systems" funded by the University of Rijeka, Croatia.

\section{REFERENCES}

1. A.R.S. Bahai, B.R. Saltzberg, Multi-Carrier Digital Communications - Theory and Applications of OFDM, Kluwer Academic Publishers, USA, 1999.

2. F. Bekkadal, Emerging Maritime Communications Technologies, 9th International Conference on Intelligent Transport Systems Telecommunications, (ITST), pp. 358-363, 2009.

3. R.W. Chang, Synthesis of Band-Limited Orthogonal Signals for Multichannel Data Transmission, Bell System Technical Journal, 45, pp. 1775-1796, 1966.

4. K. Dhunes, An Offset Modulation Method Used to Control the PAPR of an OFDM Transmission, $\mathrm{PhD}$ dissertation, University of Pretoria, Faculty of Engineering, Built Environment and Information Technology, South Africa, 2012.

5. M. Engels, Wireless OFDM Systems: How to Make Them Work?, The Springer International Series in Engineering and Computer Science, USA, 2002.

6. K. Fazel, S. Kaiser, Multi-Carrier and Spread Spectrum Systems, John Wiley \& Sons, United Kingdom, 2008. 
7. S. Gajewski, Design of OFDM-based radio communication systems for coast-to-sea and coast-to-air propagation environments, Polish Maritime Research, 1(89), Vol. 23, pp. 12-19, 2016.

8. A.A. Giordano, A.H. Levesque, Modeling of Digital Communication Systems Using SIMULINK, John Wiley \& Sons, Inc., Hoboken, New Jersey, 2015.

9. D. Green et al., VHF Propagation Study, Defence Research and Development, Canada, 2011.

10. B. Hui et al., Design of radio transmission technologies for $V H F$ band ship ad-hoc network, International Conference on ICT Convergence (ICTC), Seoul, pp. 626-629, 2011.

11. ITU, Recommendation ITU-R M.1842-1, Characteristics of $V H F$ radio systems and equipment for the exchange of data and electronic mail in the maritime mobile service $R R$ Appendix 18 channels, Electronic Publication, Geneva, 2009.

12. ITU, Recommendation ITU-R M.1798-1, Characteristics of $H F$ radio equipment for the exchange of digital data and electronic mail in the maritime mobile service, Electronic Publication, Geneva, 2010.

13. ITU, Report ITU-R M.2287-0, Automatic identification system VHF data link loading, Electronic Publication, Geneva, 2013.

14. ITU, IALA - Working Document toward a draft new Report Maritime Radiocommunication Systems and Requirements, Radiocommunication Study Groups, 2014.

15. ITU, Report ITU-R M.2231-1, Use of Appendix 18 to the Radio Regulations for the maritime mobile service, Electronic Publication, Geneva, 2014.

16. M.C. Jeruchim, P. Balaban, K.S. Shanmugan, Simulation of Communication Systems: Modeling, Methodology, and Techniques, Second edition, Information Technology: Transmission, Processing, and Storage, Kluwer Academic Publishers, USA, 2002.

17. L. Kansal, A. Kansal, K. Singh, Analysis of Different High Level Modulation Techniques for OFDM System, International Journal of VLSI and Signal Processing Applications, 1(2), pp. 102-107, 2011.

18. S.G. Kim et al., Design and Implementation of a $\pi / 4-D Q P S K$ Transmitter for Maritime VHF Digital Communications, OCEANS, Spain, 2011.

19. Y.H. Kim, I. Song, H.G. Kim, T. Chang, H.M. Kim, Performance Analysis of a Coded OFDM System in Time-Varying Multipath Rayleigh Fading Channels, IEEE Transactions on Vehicular Technology, 48(5), pp. 1610-1615, 1999.
20. M. Kiviranta et al., Constant Envelope Multicarrier Modulation: Performance Evaluation in AWGN and Fading Channels, IEEE Military Communications Conference, 2, pp. 807-813, 2005

21. M.Z. Parvez, Peak to Average Power Ratio (PAPR) Reduction in OFDM Based Radio Systems, MSc Thesis, Blekinge Institute of Technology, School of Engineering, Department of Signal Processing, Sweden, 2010.

22. Y.J. Qazi, Performance Evaluation of Error Correcting Techniques for OFDM Systems, MSc Thesis, Blekinge Institute of Technology, Electrical Engineering, Sweden, 2014.

23. M.I. Rahman, S.S. Das, F.H.P. Fitzek, OFDM Based WLAN Systems, Technical Report R-04-1002, Aalborg University, 2005.

24. H. Rohling, OFDM - Concepts for Future Communication Systems, Springer, Germany, 2011.

25. H. Schulze, C. Lüders, Theory and Applications of OFDM and CDMA - Wideband Wireless Communications, John Wiley \& Sons Ltd., England, 2005.

26. W. Shieh, I. Djordjevic, Orthogonal Frequency Division Multiplexing for Optical Communications, Elsevier, USA, 2010.

27. S. Shooshtary, Development of a MATLAB Simulation Environment for Vehicle-to-Vehicle and Infrastructure Communication Based on IEEE 802.11p, MSc Thesis, University of Gävle, Department of Technology and Built Environment, Vienna, Austria, 2008.

28. C.Y.D Sim, The propagation of VHF and UHF radio waves over sea paths, $\mathrm{PhD}$ dissertation, University of Leicester, Department of Engineering, United Kingdom, 2002.

29. M.K. Simon, M.S. Alouini, Digital Communication over Fading Channels - A Unified Approach to Performance Analysis, John Wiley \& Sons, NY, 2000.

30. G.L. Stuber, Orthogonal Frequency Division Multiplexing for Wireless Communications, Springer, USA, 2006.

31. V. Tarokh, New Directions in Wireless Communications Research, Springer, USA, 2009.

32. World Radiocommunication Conference (WRC-12), RESOLUTION 359, Consideration of regulatory provisions for modernization of the Global Maritime Distress and Safety System and studies related to e-Navigation, Geneva, 2012.

33. World Radiocommunication Conference (WRC-12). RESOLUTION 360, Consideration of regulatory provisions and spectrum allocations for enhanced Automatic Identification System technology applications and for enhanced maritime radiocommunication, Geneva, 2012. 
34. F. Xiong, Digital Modulation Technique: Second edition, Artech House Inc., Norwood, 2006.

\section{CONTACT WITH THE AUTHORS}

Sanjin Valčić

e-mail: svalcic@pfri.hr

Faculty of Maritime Studies Rijeka, University of Rijeka, Studentska 2, 51000 Rijeka

Croatia

Tibor Pogány

e-mail:poganj@pfri.hr

Faculty of Maritime Studies Rijeka, University of Rijeka, Studentska 2, 51000 Rijeka

Croatia

John von Neumann Faculty of Informatics, Institute of Applied Mathematics, Óbuda University, Bécsi út 96/b, 1034 Budapest

HUNGARY

\section{Zoran Mrak}

e-mail:mrak@pfri.hr

Faculty of Maritime Studies Rijeka, University of Rijeka, Studentska 2, 51000 Rijeka

Croatia 